\title{
EDITORIAL
}

\section{Reduced-intensity conditioning allogeneic stem cell transplantation: hype, reality or time for a rethink?}

Leukemia (2006) 20, 1653-1654. doi:10.1038/sj.leu.2404336

In selected patients, conventional standard myeloablative allogeneic stem cell transplantation (allo-SCT) is a curative treatment modality, especially in patients with myeloid leukemia. However, it is well established that the overall benefit of myeloablative allo-SCT can be offset by a significantly high rate of procedure-related toxicity, morbidity and transplant-related mortality. Thus, conventional myeloablative allo-SCT is rarely considered for elderly patients, or patients with comorbidities. Also, diagnoses such as lymphoma or myeloma are usually considered as a contra-indication for the use of myeloablative allo-SCT. In the last decade, the so-called non-myeloablative or reduced-intensity conditioning (RIC) regimens for allo-SCT have emerged as an attractive modality to decrease allo-SCT-related toxicities. Indeed, RIC allo-SCT represents an attempt to harness the well-documented immune graft-versus-tumor (GVT) effect while attempting to control or overcome toxicity. The work of different pioneering groups rapidly proved that this approach is feasible in several disease settings or patients' categories, and had the added benefit of expanding the transplant option to patients who are ineligible for myeloablative allo-SCT. Currently, the use of RIC allo-SCT has challenged the need for highdose conditioning regimens. However, one should bear in mind that the RIC allo-SCT approaches were not developed with the same group of patients in mind. On the one hand, RIC and highly immunosuppressive regimens may be viewed with great interest as the basis for subsequent adoptive immunotherapy in patients whose tumors are known to be chemoresistant (e.g. metastatic solid tumors). On the other hand, other groups of patients viewed as classical indications for allo-SCT (e.g. acute or chronic myeloid leukemia) may clearly benefit from a decreased rate of toxicity, especially those elderly patients not regarded eligible for the conventional allo-SCT procedure, most commonly because of advanced age, comorbidities or toxicities from preceding therapies.

Unfortunately, and despite several thousands of patients receiving RIC allo-SCT reported to national and international registries, the true value of RIC allo-SCT in the management of hematological and non-hematological malignancies is, as yet, difficult to delineate. Several reasons can help understanding these difficulties. A consistent definition of 'non-myeloablative' or 'RIC' regimens is still lacking with respect to drug classes, doses and durations. Thus far, multiple so-called RIC regimens have been used, ranging from low-dose total-body irradiation (2 Gy) plus immunosuppressive agents such as mycophenolate mofetil and cyclosporin A to regimens using combinations of chemotherapy and in vivo T-cell-depleting and immunomodulatory agents such as antithymocyte globulin, or even myeloablative drugs in doses similar to standard allo-SCT. Thus, these $\mathrm{RIC}$ regimens comprise a continuum that overlaps with standard myeloablative regimens making it difficult to draw firm conclusions. This may be crucial in diseases such as acute leukemia where the fear would be that, by reducing the intensity of the antileukemic chemotherapy, the overall benefit of the allo-SCT procedure would be reduced, unless new tools are used to rapidly induce the immunologic GVT effect. The latter raises the issue of graft-versus-host disease (GVHD), where in theory, reduction of the 'inflammatory' component of the conditioning as in RIC or non-myeloablative regimens may lead to the reduction of the rate and/or severity of GVHD. However, such hypothesis is yet to be proven, at least in clinical practice, where GVHD remains a matter of concern after RIC allo-SCT. Moreover, the distinction between acute and chronic forms of GVHD seems to be blurred after RIC allo-SCT, with clinically 'acute' forms occurring much later than usual, or clinically 'chronic' forms occurring very early, raising questions about the need for continuous immunosuppression and its corollary of long-term infectious complications. Also, the notion or definition of 'ineligibility' for conventional standard allo-SCT is not clearly defined. Efforts are currently ongoing in several groups to establish scoring systems, based on patient age, previous medical history and comorbidities to clearly identify those patients 'ineligible' for standard allo-SCT. However, the concept of 'ineligibility' may also change over time with the introduction of new anti-infectious agents (e.g. new anti-fungal drugs) or less toxic forms of chemotherapeutic agents (e.g. oral versus intraveneous busulfan).

Currently, there are no prospective, randomized or controlled trials that addressed the specific role of RIC allo-SCT in net health outcomes that should include, in a specific disease setting, an analysis of disease-free survival and overall survival balanced against treatment-related toxicity, complications and death. Most of the studies, although some occasionally discussed their results in reference to outcomes of standard allo-SCT, reported case series, most of which lacked concurrent or historical controls and in which outcomes of interest were not uniformly reported. These studies are very heterogeneous, largely because they included a 'mixed bag' of treatmentspecific outcomes, and combined patients with several diseases in the same analysis, and differed in patient selection criteria, RIC regimens and timing of RIC allo-SCT (after autologous transplant, in first or second remission, after relapse, or in refractory conditions), making conclusions very limited from the overall body of evidence in this area. Similarly, quality of life data and data on the specific nature of comorbid conditions that motivated the use of RIC allo-SCT are usually scant, and the number of patients who had undergone RIC allo-SCT with comorbidities is unclear in many studies.

At present, the optimism to regard RIC allo-SCT as a potential and promising treatment modality for many patients remains very high among investigators. However, the enthusiasm for a 'one size fits all' RIC allo-SCT approach has waned and is far from a cure to many diseases, highlighting the need for a renewed clinical and therapeutic research in this area.

With this background, Leukemia will publish a series of Spotlight articles (first part of which to be published with this issue) focused on the use of RIC allo-SCT. These articles will assess the current status of RIC allo-SCT in specific disease 
settings (AML, MDS, myeloma, lymphoma, etc.), but aspects related to the diversity of $\mathrm{RIC}$ regimens and transplant-related complications (GVHD, infections, immune recovery, etc.) will also be addressed. Experts in the field were asked not to write a remake of previous studies, but rather to synthesize the available research evidence, to expose the pros and cons in a lively debate, to provide their own opinion and a very thorough, indepth picture of the problem allowing for contradictions. We hope that such articles will pave the way for a number of collaborative efforts and controlled clinical trials aiming to establish definitely the exact role of RIC allo-SCT in the therapeutic armamentarium, making it less hype and more reality.

M Mohty ${ }^{1}$, A Nagler ${ }^{2}$ and NM-B Killmann ${ }^{3}$ ${ }^{1}$ Unité de Transplantation et de Therapie Cellulaire, Institut Paoli-Calmettes, Marseille, France;

${ }^{2}$ Division of Hematology, Chaim Sheba Medical Center, Tel Hashomer, Israel and

${ }^{3}$ Editor-in-Chief, Leukemia, 26 rue Miollis, Paris, France E-mail:mohtym@marseille.fnclcc.fr 